\title{
Experiment DsTau (NA65) - study of tau neutrino production at CERN SPS
}

\section{S. Vasina* for the DsTau (NA65) collaboration}

Joint Institute for Nuclear Research, Joliot-Curie 6, Dubna, Russia

E-mail: vasina@jinr.ru

The goal of the DsTau (NA65) experiment at CERN SPS is the measurement of tau neutrino production. The dominant source of tau neutrinos is decay of $D_{s}$ mesons, produced in protonnucleon interactions, to tau neutrino and tau lepton with further decay of tau lepton. Thus, the experiment aims to measure differential cross section of this reaction. This measurement will allow to re-evaluate DONuT results reducing the systematic error in tau neutrino interaction cross section caused by the tau neutrino flux uncertainty in beam dump experiments from $50 \%$ to $10 \%$. Such accuracy of cross section measurement will allow testing the Lepton Flavour Universality for neutrinos. The experiment exploits nuclear emulsion detectors with $\mu \mathrm{m}$ space resolution allowing to recognize the peculiar $D_{s}$ to $\tau$ decay topology in a few $\mathrm{mm}$ range. In addition, about $10^{5}$ charmed particle decays are expected in the experiment, which makes possible to study charm physics, in particular to search for intrinsic charm component in proton. About $10 \%$ of whole data were collected during 2018 pilot run and analysis of this sample is ongoing. Given the relevance of the study and encouraging results of the data analysis, CERN had approved the DsTau project as a new experiment NA65 in 2019. The main data sample will be collected in 2021-22 physics runs. In this paper, the status and prospects of NA65 as well as the results of the pilot run are presented.

40th International Conference on High Energy physics - ICHEP2020

July 28 - August 6, 2020

Prague, Czech Republic (virtual meeting)

\footnotetext{
${ }^{*}$ Speaker
} 


\section{Introduction}

The existence of tau neutrino was predicted after the tau lepton discovery in 1975 [1] and it was detected only 25 years later in the DONuT [2] experiment. Still properties of $v_{\tau}$ are not well studied due to quite limited measurements. In particular, the cross section of tau neutrino charged current (CC) interaction is known with a large statistical and systematic uncertainties in comparison with other neutrino flavours. The cross section measurement with a better accuracy would allow testing the Lepton Flavour Universality (LFU) of Standard Model in neutrino interactions. Several results for B-meson decay asymmetry ( $\mathrm{LHCb}, \mathrm{Babar}$, Belle) demonstrate hints of possible LFU violation that may be related to Physics Beyond Standard Model effects.

So far, the tau neutrino interaction cross section was measured by DONuT [3], OPERA [4] and SK [5] experiments under rather different conditions, which make the results difficult to compare. In general, all the measurements have large statistical and/or systematic errors of 30-50\% caused by poor statistics and experimental uncertainties. The future beam-dump experiments, for example, SHiP, could provide measurements with negligible statistical errors [6]. Thus, the overall accuracy of the cross section in such experiments will be determined by the systematic errors, in particular, by the $v_{\tau}$ flux uncertainty, which is to be improved in the DsTau (NA65) experiment.

In the accelerator-based neutrino beam source of $v_{\tau}$ is mainly decay of $D_{s}^{ \pm}$mesons produced in proton-nucleus interactions: $D_{s}^{-} \rightarrow \tau^{-} \bar{v}_{\tau}$ with the further decay $\tau^{-} \rightarrow v_{\tau} X$. Such $v_{\tau}$ beam has rather large divergence due to the emission angle of $D_{s}$ and to the decay momenta. Thus, calculation of the tau neutrino flux on the detector requires the knowledge of the differential production cross section of $D_{s}$. Although there were several measurements on charmed particles, there is a lack of measurements on the $D_{s}$ differential production cross section in the proton interactions, which contributes the main uncertainty of the $v_{\tau}$ cross section measurements $(>50 \%)$. Thus, a new measurement of the differential production cross section of $D_{s}$ is quite important for future neutrino experiments as well as for re-evaluation of the DONuT results.

The DsTau (NA65) experiment studies $v_{\tau}$ production by measuring $D_{s} \rightarrow \tau$ decays in protonnuclear interactions at CERN SPS. DsTau (NA65) exploits nuclear emulsion techniques. Its high spacial resolution allows recognizing peculiar "double-kink" topology of $D_{s} \rightarrow \tau$ decays. The project aims to register $\sim 1000$ such decays in $2.3 \times 10^{8}$ proton interaction in the tungsten target. This goal can be achieved by using of nuclear emulsion, nanometric-precision readout and highspeed automatic scanning stations developed in the last two decades. DsTau (NA65) will provide an independent $v_{\tau}$ flux prediction for the future neutrino beams with an accuracy below $10 \%$. Then, the $v_{\tau} \mathrm{CC}$ cross section measurement with sufficiently low systematic uncertainty will allow testing LFU in neutrino interactions.

In addition to the main goal, DsTau (NA65) is able to study charm physics, since $\sim 10^{5}$ charmed particle pairs are expected in $2.3 \times 10^{8}$ proton interaction. The analysis of these events could allow measuring the intrinsic charm component in proton [7] by measuring the emission angle (pseudorapidity) of the charmed particle pairs, the interaction length of charmed hadrons, the $\Lambda_{c}$ production rate and search for super-nuclei [8]. 


\section{Experiment overview}

The $D_{s} \rightarrow \tau$ decays have a very peculiar topology, the decay has "double-kink" and there is a charge or neutral pair charm in the interaction vertex, Fig. 1. However, reconstruction of such events is quite challenging because of short flight length of $D_{s}$ and kink angle of tau lepton is a few mrad. The measurement of $D_{s}$ momentum is difficult as well due to presence of two $v_{\tau}$, which are not detected in the detector. Therefore, the use of emulsion detector with nanometric spatial resolution allows identify such decay topologies. The emulsion films provide a position resolution of about $50 \mathrm{~nm}$ and an angular resolution of $0.34 \mathrm{mrad}$.

The main source of background is interactions of secondary hadrons (the products of primary proton interactions) with no nuclear fragments detected. Based on the FLUKA [9] simulation, the number of expected background events for the full data sample is about 18.

The basic unit of the DsTau detector is shown in Fig. 1. It consists of 0.5-mm-thick tungsten target followed by 10 emulsion plates interleaved with 9 plastic sheets with thickness of $200 \mu \mathrm{m}$. Each emulsion plate consists of a $210 \mu \mathrm{m}$ plastic base with $70 \mu \mathrm{m}$ emulsion layers on both sides. The combination of 10 basic units form a decay module. It is followed by so called Emulsion Cloud Chamber (ECC), which is made of $261-\mathrm{mm}$-thick lead plates interleaved by emulsion plates. ECC is used for charged particle momentum measurement. Several emulsion plates with plastic separators in the front side of the detector serve as beam protons tagger. The total size of detector is $12.5 \times 10.0 \times 8.6 \mathrm{~cm}$.

A schematic view of experimental setup is shown in Fig. 1. The beam profile is measured by a silicon pixel telescope and time profile is measured by a scintillation counter. The detector is driven by a target mover along $\mathrm{X}$ and $\mathrm{Y}$ axes according to counter signal to have uniform distribution of primary protons with about $10^{5}$ tracks $/ \mathrm{cm}^{2}$ in the detector.

The analysis of the track information accumulated in the emulsion during exposure is done in two steps. First, scanning of the full emulsion area by a fast system, so called Hyper Track Selector (HTS) [10]. HTS has a relatively coarse angular resolution $(\sim 2.5 \mathrm{mrad})$, and detects events, which have two decays in a short distance, namely the decays of $\tau$ and partner charm $\left(D^{ \pm}\right.$or $\left.D^{0}\right)$. Second step is a high precision measurement around the $\tau$ decay candidates to find $D_{s} \rightarrow \tau$ small kinks. For this step another scanning microscope with a slower readout speed, but with a high angular resolution of $0.16 \mathrm{mrad}$ is used. More details on the scanning systems are given in [11, 12].

\section{Reconstruction and analysis}

The automatic scanning systems read and digitize the track information which is similar to the case of any electronic detector. The output of the readout is the information on the track segments at both sides of the emulsion film. These two segments are linked together using the so called basetrack, which is a basic unit of information used for the further track reconstruction. The readout is completed for the whole data sample collected in 2018 pilot run.

The basic idea of the track reconstruction is a linking of basetracks at the different films according to their angles and positions. In DsTau this task is quite challenging due to high track density of $10^{5}-10^{6}$ tracks $/ \mathrm{cm}^{2}$. Nevertheless, the unique spacial emulsion capabilities and a new tracking algorithm [12] allow to reconstruct basetracks efficiently. The averaged basetrack finding 
Figure 1: Schematic view of the "double-kink" event in the basic unit of DsTau decay module (left) and scheme of the experimental setup (right) [12].

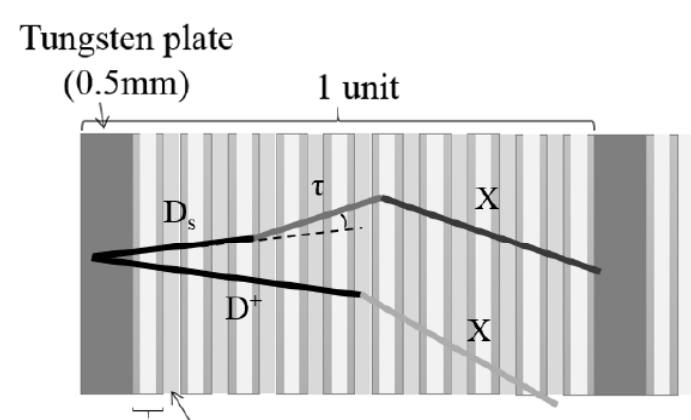

Emulsion film Plastic sheet $(200 \mu \mathrm{m})$

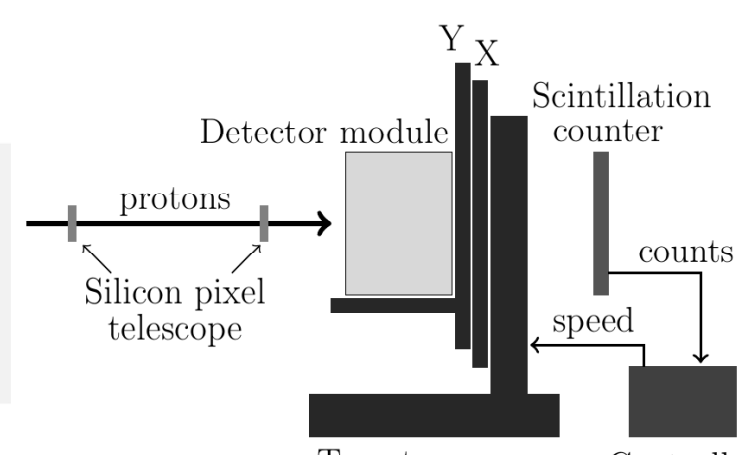

Target mover

Controller

Figure 2: The film-by-film basetrack finding efficiency(left) and the evolution of track density as a function of depth in a module (right) [12].
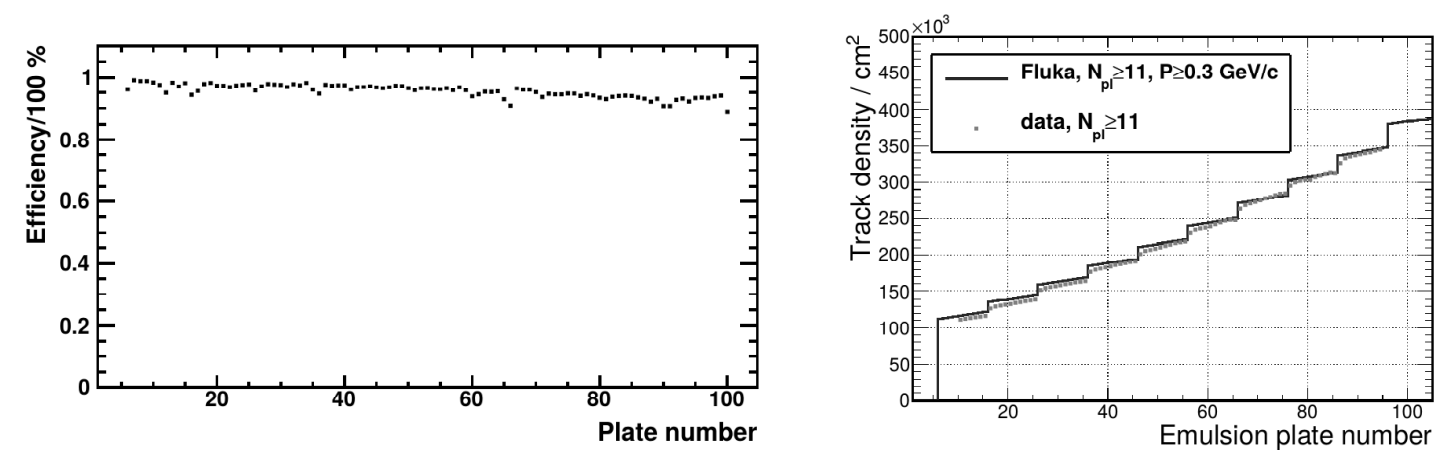

efficiency is higher than 95\%, shown in Fig. 2. It shows a slow decrease towards the downstream part of the detector due to increase of the track density, but it is still high enough to reconstruct tracks with an efficiency of $>99 \%$.

The reconstructed tracks are used to reconstruct vertices, and a systematic search for a decay topology of short-lived particles is applied. The statistics of the found vertices and events with the double decay topology observed in a sub-sample of the data are shown in Table 1. It is consistent with the expectation based on the simulation for the equivalent data sample.

Table 1: Statistics found in the sub-sample of 2016, 2018 data related to $>34.2 \times 10^{6}$ protons analyzed.

\begin{tabular}{|c|c|c|c|}
\hline & Observed & \multicolumn{2}{|c|}{ Expected } \\
\hline Vertices in tungsten & 147,236 & \multicolumn{2}{|c|}{155,135} \\
\hline & & Signal & Background \\
\hline Double decay topology & 115 & $80.1 \pm 19.2$ & $12.7 \pm 5.0$ \\
\hline
\end{tabular}


Figure 3: Flight length distributions for charged 1-prong and neutral 2-prong decay candidatesin the doublecharm event samples. The FLUKA MC histograms are area-normalized to data.
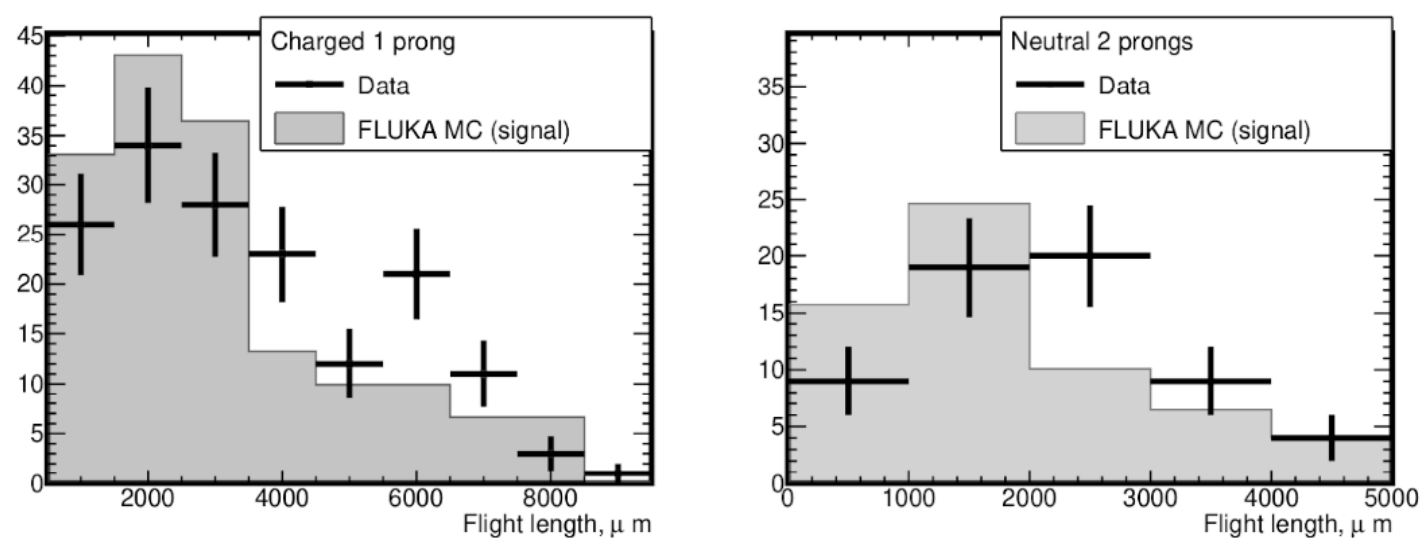

\section{Conclusion and prospects}

The DsTau experiment studies the tau neutrino production in $400 \mathrm{GeV} / \mathrm{c}$ proton interactions with the tungsten target by measuring the differential production cross-section of $D_{s}$ mesons, which is important for future neutrino experiments. The collected data allows to study charm physics in proton interactions with a high statistics.

DsTau performed test runs in 2016 and 2017 and successfully collected about $10 \%$ of full statistics during 2018 pilot run. The experiment was approved by CERN SPSC as NA65 and the physics runs are planned for 2021-22 years. The analysis of the data sample collected in the pilot run as well as preparations to the physics run are ongoing successfully.

\section{Acknowledgments}

We thank for the support of the beam physicists at CERN for the successful data taking in the proton beam at the SPS north area. We would like to acknowledge the precious contributions of the technical staff from LHEP Bern. We acknowledge N. Naganawa and the colleagues from Nagoya University for sharing the expertise of emulsion detector. We warmly thank students from Middle East Technical University and Nagoya University who participated in the beam exposure campaign.

This work was supported by JSPS KAKENHI Grant Numbers JP 18KK0085, JP 17H06926, and Konica Minolta Science and Technology Foundation Research Grant for Photographic Science and project No. 16 (Study of the tau neutrino production in proton-nucleus interactions) between JINR, Dubna, Russia and ISS, Romania. This work was partially supported by the Swiss National Science Foundation Ambizione grant PZ00P2_154833, especially on the detector R\&D. We also appreciate the support from the TAEK of Turkey and JINR grant for young scientists 20-202-02.

\section{References}

[1] M. Perl et al., Evidence for Anomalous Lepton Production in $e^{+} e^{-}$Annihilation, Phys. Rev. Lett. 35 (1975) 1489. 
[2] K. Kodama et al.(DONuT Collaboration), Observation of tau neutrino interactions, Phys. Lett. B 504 (2001) 218-224, [hep-ex/0012035].

[3] K. Kodama et al. (DONuT Collaboration), Final tau-neutrino results from the DONuT experiment, Phys. Rev. D 78 (2008) 052002.

[4] N. Agafonova et al.(OPERA Collaboration), Discovery of $\tau$ Neutrino Appearance in the CNGS Neutrino Beam with the OPERA Experiment, Phys. Rev. Lett. 115 (2015) 121802 [hep-ex/1507.01417v2]

[5] Z. Li et al. (Super-Kamiokande Collaboration), Measurement of the tau neutrino cross section in atmospheric neutrino oscillations with Super-Kamiokande, Phys. Rev. D 98 (2018) 052006 [hep-ex/1711.09436].

[6] M. De Serio (SHiP Collaboration), Neutrino physics with the SHiP experiment at CERN, PoS EPS-HEP2017 (2017) 101.

[7] Weidong Bai, Mary Hall Reno, Prompt neutrinos and intrinsic charm at SHiP, JHEP 02 (2019) 077 [hep-ph/1807.02746].

[8] V.V. Lyukov, Study of hypernuclear production and search for supernuclei in proton-nuclear interactions in photoemulsion at 70 and $250 \mathrm{GeV}$, Nuovo Cim. A 102 (1989) 583-595.

[9] T. T. Böhlen., F. Cerutti, M. P. W. Chin, A. Fasso, A. Ferrari, P. G. Ortega, A. Mairani, P. R. Sala, G. Smirnov and V. Vlachoudis, The FLUKA Code: Developments and Challenges for High Energy and Medical Applications, Nucl. Data Sheets 120 (2014) 211.

[10] M. Yoshimoto, T. Nakano, R. Komatani, H. Kawahara, Hyper-track selector nuclear emulsion readout system aimed at scanning an area of one thousand square meters, PTEP 10 (2017) 103 [physics.ins-det/1704.06814v3].

[11] S. Aoki, et al., Experimental Proposal, Study of tau-neutrino production at the CERN-SPS, CERN-SPSC-2017-029, SPSC-P-354 [hep-ex/1708.08700].

[12] S. Aoki et al. (The DsTau/NA65 Collaboration), DsTau: Study of tau neutrino production with $400 \mathrm{GeV}$ protons from the CERN-SPS, JHEP 01 (2020) 033 [hep-ex/1906.03487v1] 